\title{
The Mechanisms of Neuronal Interaction Regulation Mechanisms
}

\author{
N. P. Vesselkin \\ Sechenov Institute of Evolutionary Physiology and Biochemistry, Russian Academy of Sciences, St. Petersburg; \\ e-mail:npves@yahoo.com
}

DOI: $10.1134 / \mathrm{S} 1990747809030490$

It is well known that synaptic transmission is the main neuronal interaction mechanism representing a specialized chemical signaling. The interaction of two neurons can be regulated at the pre- and postsynaptic levels. Using the electron microscopic immunocytochemistry it was shown that the axons making synapses on the vertebrate motoneurons are contacted not only by GABAergic, but also by glutamate- and glycine-containing terminals. It testifies that multiple neurotransmitters can be used for presynaptic regulation of synaptic transmission. This statement is confirmed by postsynaptic potential and current modifications after membrane receptor agonist and antagonisat application. The co-release of different transmitters and postsynaptic effect interactions are considered as possible mechanisms of synaptic transmission regulation.

The work was supported by the Russian Foundation for Basic Research (project no. 08-04-00098a) and by the Program Physiologycal Mechanisms of Regulation of Internal Milieu and Living System Behavior of the RAS Department of Biological Sciences. 